the way the decision will go - and the decision then indeed goes this expected way and ends there.

Three sets of interpretive 'formulas' are examined. First, in the chapter entitled 'broad', formulas are examined that embody either broad or restrictive interpretation. Which formulas exist in the body of case-law examined that embody a broad or restrictive approach? In which circumstances are they typically used? How do they evolve? When are they decisive for the Court's decision, when do they provide impetus, exert 'spin'? These are the questions that are answered first for 'broad' interpretation. The chapter on 'broad' interpretive formulas is different from the other two chapters on interpretive formulas in that it may contain aspects that are relevant beyond the law of the Union and the Court of Justice. Broad or restrictive interpretation is common in other legal orders, too, like in international and domestic law. Yet, these broader implications and possible cross-connections are not addressed in this book, because it is about the case-law of the Court of Justice. In the next chapter the above questions are pondered for interpretive formulas in which the Court relied on the idea that parts of Union law merely coordinated national law, as an alternative to harmonizing it. This is the chapter entitled 'coordinated'. This interpretation is highly specific for the law of the Union and the case-law of the Court. Coordination of legal systems is - at least in this terminology and as far as can be judged - unique to the law of the Union. Within this law, social security is of primary importance. The final chapter labelled 'fundamental' ponders the above questions for formulas drawing on the idea that some notions are fundamental, while others are not. Like broad interpretation, interpretation relying on hierarchy, such as a notion being fundamental, is not unique to the law of the Union. Most legal orders, perhaps even all, at one point or another prioritize some notions over others. However, this chapter is uniquely tied to the law of the Union in that it primarily deals with Union citizenship and an intepretive formula used exclusively with regard to it ('the fundamental status').

\title{
III Why is the first part on 'the case-law' necessary? Why not cover more interpretive formulas?
}

Why not drop the voluminous first part on 'the case-law' and cover more interpretive formulas? And why are three interpretive formulas investigated and others left aside? Answers to these questions are not easy to give. They to some degree concern scientific honesty and, in addition, require a deep understanding of the case-law that is the subject of the first part of this book. Admittedly, the evolution of further interpretive formulas could be traced. For instance, the formula used to argue that social security rights based solely on national law must not be lost after the right of free movement has been exercised would have been an alternative; or the formula relying on the need for cohesion of the tax system. 
However, several points are important in this regard. First, to live up to scientific rigour the first part on 'the case-law' is indispensable. The body to be investigated must first be established - the facts, the data must first be collected and described, so to speak - before it can be discussed. A crucial step would be missed, if discussion and interpretation were begun right away. Uncertainty about the framework and the limits of the interpretation would result, ultimately rendering the study useless. Second, it cannot be avoided to be selective when it comes to interpretive formulas. Dozens of interpretive formulas are used in the body of case-law under scrutiny, a body of case-law that is, moreover, exceptionally large. It would fill many more volumes, if all of those formulas were to be traced. Third, given the need to select, variety is important. Hence, interpretive formulas are chosen that shed light on as many different aspects of the case-law as possible. The broad as well as the restrictive formulas discussed first in the second part elucidate the Court's activism to some extent and are potentially important beyond the law of the Union; the formula of coordination, which comes next, illuminates a possibly unique structural element in the Court's case-law; and the fundamental formula(s) partly reveal(s) how the case-law relies on hierarchy, in particular with regard to an 'institution' that is central to the Union, i. e. Union citizenship. The examination of this set of formulas, hopefully, allows us to achieve at least a better understanding of the Court's case-law in persons and services and of the way it evolves. Fourth, quite plainly, those interpretive formulas are traced in the case-law which, after years of careful study of the case-law, turned out to be most interesting. A personal element is, obviously, involved in this choice. But it is an informed choice and one the reader hopefully finds plausible.

\section{A text-based approach}

For all three interpretive formulas the analysis is based on the text of the decisions of the Court. This has two consequences. First, it is possible that some decisions escape scrutiny which, generally speaking, turn out to have a broad, sweeping character or implicitly rely on a coordinative or fundamental approach without that this is reflected in the wording of the decisions. Second, this book is not capable of fully answering the question why a specific formula is used in a decision. Factors outside the text of a decision influence the answer to the question 'why'. These factors include the composition of the Court, the opinion of the Advocate General, or the person who writes the decision. Such factors are not taken into account in this book because of the text-based approach. The book rather explains the 'when', the 'how', and to a certain extent the 'wherefore' for the relevant formulas. Yet, although the 'why' is ignored, the contribution this book makes is significant. As indicated above, the analysis of 'broad' formulas clarifies an aspect of the Court's activism, at least implicitly; the en- 